\title{
The role inflammatory response genes in obstructive sleep apnea syndrome: a review
}

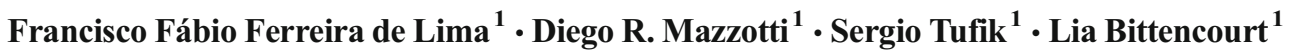

Received: 3 March 2015 / Revised: 21 June 2015 / Accepted: 29 June 2015 / Published online: 23 July 2015

(C) Springer-Verlag Berlin Heidelberg 2015

\begin{abstract}
Background Obstructive sleep apnea syndrome (OSAS) has a negative impact on health and behavior of millions of individuals worldwide. The pathogenesis of this disorder is a multifactorial process related to a variety of mechanisms, including selective activation of inflammatory response pathways. A number of inflammatory factors, such as IL-6, IL-8, and TNF- $\alpha$, can be found in high concentrations in subjects with OSAS and may serve as biological markers of this disease. The concentration of these cytokines contributes to weight gain in patients with OSAS and can also modify the risk of obesityrelated metabolic disorders, especially insulin resistance. Nevertheless, the mechanisms by which specific genes are associated with these processes are still poorly known. In addition to gene expression studies, investigations aiming at the identification of epigenetic factors associated with OSAS are still scarce in the literature. The documented data support the hypothesis that the molecular changes that mediate inflammatory response are important mechanisms in the pathogenesis of OSAS, sleepiness, insulin resistance, visceral obesity, and cardiovascular disease, perhaps by leading to a more severe OSAS. Often, systemic changes may not be detected in mild OSA; however, molecular changes, which are much more sensitive to the mechanisms of intermittent hypoxia and oxidative stress, may be present.

Purpose This review aimed to show an updated view on the studies evaluating the genetic basis of inflammatory response
\end{abstract}

Diego R. Mazzotti

mazzottidr@gmail.com

1 Departamento de Psicobiologia, Universidade Federal de São Paulo, Rua Napoleão de Barros, 925 Vila Clementino, São

Paulo, SP 04024-002, Brazil in many aspects of OSAS and to highlight potential research areas not fully explored to date in this field.

Keywords Inflammatory markers · Obstructive sleep apnea · Gene expression

\section{Introduction}

\section{Obstructive sleep apnea syndrome}

The obstructive sleep apnea syndrome (OSAS) is characterized by episodes of partial or total upper airway obstruction during sleep with airflow interruption (apnea) or reduction (hypopnea) leading to transient reduction in oxyhemoglobin saturation, hypercapnia, followed by a transient awakening, which leads to the reestablishment of the upper airway permeability. Consequently, the patient generally presents fatigue and excessive daytime drowsiness, attention or memory impairment, and habitual snoring history, being the latter an indicative of increase of the airway flow resistance in the pharynx [1,2].

The number of apnea/hypopnea events during sleep can be characterized by the apnea-hypopnea index (AHI), which measures how many events occur per hour of sleep. The repetitive occurrence of these events contributes to fragmented sleep and, as a consequence, excessive daytime sleepiness [3].

The American Academy of Sleep Medicine considers OSAS severity by the AHI classification (mild -5 to 15 ; moderate15 to 30 , and severe $\longrightarrow 30$ ) and by the degree of daytime drowsiness (mild: unwanted drowsiness or involuntary episodes occurring during activities which require little attention; moderate: during activities which require some attention, such as meetings; and severe: during activities which require more active attention, such as those during conversation or driving) [4]. 
It has been estimated that OSAS affects $\geq 4 \%$ of men and $\geq 2 \%$ of women in the world, where one in every five adults suffers from mild OSAS and one in every 15 presents mild to severe OSA [1, 5-7]. Besides the global estimate mentioned above, a cohort study by Young et al. already described a prevalence of sleep-disordered breathing by $9 \%$ in women and $24 \%$ men [7]. Also, a recent study by Peppard et al. found increased prevalence of moderate to severe OSA [8]. Our group conducted a large OSA study in a South American metropolitan area (São Paulo, Brazil) and found a higher prevalence of OSA (32.8 \%) than what was found earlier [9]. Thereby, the importance of the syndrome, which had been underestimated for a long time, is significant since it occurs in a large portion of the population [1]. The large frequency of non-diagnosed OSAS cases leads to awareness in the scientific community; once even the mild cases of OSAS, with or without daytime symptoms, could be associated with a considerable increase in cardiovascular morbidity [10].

Pathogenesis of OSAS varies, and predisposing factors include small upper airway lumen, unstable respiratory control, low arousal threshold, small lung volume, and dysfunctional upper airway dilator muscles [11]. The major OSAS risk factors are age, male gender, and obesity (higher body mass index $[\mathrm{BMI}]$ ), leading to a larger neck circumference (NC). It has already been defined that obesity is an important risk factor to OSAS, and the moderate reduction of body weight has been shown to decrease its severity [12]. The fat deposit around the pharyngeal area might be responsible for the upper airway (UA) collapse, and the abdominal fat deposit leads to the reduction of respiratory functional residual capacity and reduction of the protective mechanism of the upper airway caudal traction $[10,13]$. The increase in body weight is associated with worsening of OSAS severity, being more evident in men when compared to women. Therefore, it is understood that central obesity constitutes an important factor to the OSAS presence and progression [12].

The OSAS is more common in men than that in women, once they present a longer UA (pharyngeal portion) and larger fat deposit around it. This gender difference is already seen in early adolescence, and testosterone could be one of the main factors [14]. In women, the hormonal state has an important role in the OSAS genesis, since the prevalence of the syndrome in the postmenopausal phase is higher than before the menopause $[12,15]$. Another relevant factor in women at postmenopausal phase is the pharyngeal length, which contributes to UA collapse [16]. Also, androgens have an important role in the OSAS pathogenesis in obese women with polycystic ovary syndrome, in relation to obese women without this disorder [12, 17].

\section{Hypoxia, oxidative stress, and inflammatory response}

It is known that the OSAS pathogenesis is related to a multifactorial process with a diversity of mechanisms, including the sympathetic nervous system hyperactivity, oxidative stress, vascular endothelial dysfunction, metabolic deregulation, and selective activation of the inflammatory airways $[18,19]$. Exposure to intermittent hypoxia $(\mathrm{IH})$ has shown several effects on the cardiovascular system in animals. According to Ryan et al., studies assessing IH in rats and dogs have reported a significant increase in arterial blood pressure, which is kept even after stimulus cessation. Moreover, the increased sympathetic activity and the decrease of baroreflex sensitivity have been proposed as factors contributing to the increase of blood pressure in animals [20]. In humans, Lam et al., described that the clinical manifestations in OSAS patients are associated with physiopathological consequences caused by chronic exposure to $\mathrm{IH}$, leading to cardiovascular morbidity, including systemic arterial hypertension and increased risk of cerebrovascular accident [21].

Specific extracellular stimuli, mediated by hypoxia, activate a signaling cascade which leads to an unbalanced production of reactive oxygen species (ROS - including free radicals, such as superoxide, and non-free radicals such as hydrogen peroxide) and endogenous antioxidants defense mechanisms [21, 22]. Mitochondrial dysfunction is one of the most important characteristics of hypoxia, which results in inducing ROS production via complex I of the respiratory chain [23]. The ROS activate an inflammatory cascade resulting in the increase of proinflammatory cytokines and expression of adhesion molecules [24]. According Lavie and Lavie, ROS also disrupt important signaling pathways in the arterial wall, promoting change in the inflammatory and immune functions through the activation of factor kB (NF-kB) [25]. Oxidant radicals and proteolytic enzymes affect the endothelial function during the accumulation of leukocytes and platelets in the endothelium, interacting with the vascular wall and contributing to the endothelial dysfunction, which is a subclinical condition of atherosclerosis, thus promoting the development of cardiovascular diseases [24, 26]. Leukocytes are also affected and this contributes to increased ROS production in patients with OSAS through NADPH oxidase [23].

Prolonged oxidative stress observed in non-treated OSAS reduces the activity of the endothelial nitric oxide synthase (NOS), an enzyme responsible for assisting several cells to synthesize $\mathrm{NO}$, an important neurotransmitter acting in the regulation of inflammation and autoimmune mechanisms [17, 27]. Therefore, the reduction of its production can result in endothelial dysfunction and thereby predispose to vascular morbidities in OSAS [27].

In a review regarding the molecular mechanisms of cardiovascular disease in OSAS, Lavie and Lavie reported that oxidative stress ultimately affects multiple systems by the reaction of $\mathrm{NO}$ with ROS, where oxidative stress is increased while NO is decreased, promoting inflammation and endothelial dysfunction. The authors suggest that oxidative stress may lead to arterial hypertension induced by the sympathetic activation and increased angiotensin II and endothelin-1 and can also induce 
upregulation of numerous redox status sensitive transcription factors, such as HIF-1 $\alpha$, NF-kB, sterol regulatory elementbinding proteins (SREBPs) and GATA transcription factors [23]. A recent review also addressed the relationship between OSA, oxidative stress, and intermittent hypoxia, in which reading is encouraged [28].

In 2006, Hernandez et al. reported that the plasmatic concentration of the oxidative stress marker malondialdehyde (MDA) was higher in OSAS patients than that in controls. With the intervention of nasal continuous positive airway pressure (CPAP) for 3 months, they found a significant decrease of MDA and consequently of the degree of endothelial dysfunction that predispose to vascular morbidity [29].

In this sense, it is clear that the consequences of important aspects of OSAS, especially chronic intermittent hypoxia, might activate specific pathways related to oxidative stress and inflammatory response. The understanding of such mechanisms contributed to the link between OSAS and cardiovascular disease and provides potential therapeutic approaches, with the use of positive airflow pressure devices, aiming to reduce the cardiovascular consequence of this disease.

\section{Inflammatory markers}

A common example of a biological marker of the inflammatory system is the cytokines, low molecular weight proteins produced by different kinds of cells acting as autocrine, paracrine, and endocrine modulators. Those molecules are frequently associated to the propagation of immunological response, and they are linked to specific receptors in the target cells, triggering signal transduction pathways that stimulate gene expression [30]. Also known for interacting with the sleep mechanisms in the brain, cytokines signal the central nervous system (CNS) regulating the normal sleep pattern during the alterations caused by infectious and pathological diseases [31].

In OSAS patients, several inflammatory factors can be found in high concentrations, which can be used as biological markers [32], such as interleukin-6 (IL-6) [33], interleukin-8 (IL-8) [34, 35 ], tumor necrosis factor- $\alpha(\mathrm{TNF}-\alpha)$ [35], vascular endothelial growth factor (VEGF) [36], leptine [37, 38], and C-reactive protein (CRP) [39, 40].

According to Quercioli et al., the levels of the proinflammatory cytokine TNF- $\alpha$ are correlated to the severity of OSAS and with increase of NREM sleep, leading to somnolence and fatigue. Before these evidences, the authors suggest that the OSAS can be considered as a systemic inflammatory disease [41]. According to Kang et al., there is also a relationship between sleep deprivation and cytokines. The proinflammatory cytokines, such as interleukin-1 beta (IL-1 $\beta$ ), IL-6, and TNF- $\alpha$, have been comprehensively evaluated as contributors in the regulation of sleep in the brain areas such as the hypothalamus and hippocampus [31]. The production of IL-1 is higher in the sleep onset, suggesting a variation of this cytokine in sleep-wake cycle. In addition, variation of daytime concentrations of pro-inflammatory cytokines is associated with sleep regulation, and the increase of IL- $1 \beta$ and IL-1 receptor antagonist plasmatic levels has been directly associated with sleep deprivation [42].

The literature describes that the interleukins IL-1, IL-2, IL6, IL-8, IL-18, and TNF $\alpha$ are known for increasing NREM sleep, and the interleukins IL-4, IL-10, IL-13, and TGF- $\beta$ for suppressing this stage of sleep [31]. In addition, circulating levels of IL- 6 and TNF- $\alpha$, as well as soluble adhesion molecules, such as the intracellular adhesion molecule (ICAM-1), and monocyte chemoattractant proteins (MCP-1), may be elevated in OSAS [18].

A previous study reported that VEGF blood concentration is elevated in patients with OSAS, potentially as consequence of nighttime hypoxia. This is due to the fact that sustained hypoxia $(\mathrm{SH})$ can activate transcription-mediated hypoxia inducible factor (HIF-1), resulting in an increase of the expression of a number of genes that encode some proteins, including VEGF and others such as erythropoietin (EPO), and inducible NOS. These factors ultimately mediate a directed adaptive response to hypoxia to increase tissue perfusion and oxygenation and, therefore, overcome the initial damage [43]. It is known that reoxygenation after a brief period of hypoxia experienced repeatedly and systemically in patients with OSAS may predispose to cellular stress, possibly due to mitochondrial dysfunction. Thus, it is assumed that such events promote the activation of a pro-inflammatory response mediated by $\mathrm{NF} \kappa \mathrm{B}$, a major regulator of inflammatory gene expression. The effects of activation of this factor include increased expression of proatherogenic factors such as TNF- $\alpha$, which may contribute to endothelial dysfunction and cardiovascular complications later, as previously mentioned [44].

CRP is an important serum marker of inflammation and is considered a strong indicator of cardiovascular risk and can also play a direct role in the initiation and progression of atherosclerosis. Unlike cytokines, CRP levels are stable in the same individual over $24 \mathrm{~h}$ and may reflect the degree of inflammatory response [45]. Their pro-inflammatory and proatherogenic properties were found in endothelial cells, vascular smooth muscle cells, and monocytes-macrophages, where CRP levels are also associated with oxidative stress [46].

The scientific literature describes that OSA patients have increased CRP levels [39, 47-49] where such changes, according to the study by Mills et.al.[50], occur mainly during the day due to the residual result of nocturnal arousal mediated by OSA. However, intervention studied using CPAP as a treatment for OSA showed that serum CRP levels decrease significantly, reducing therefore the risk of cardiovascular morbidity and mortality [33, 51, 52].

According to a meta-analysis carried out by Guo et.al., CPAP treatment is necessary for at least 3 months to significantly reduce CRP levels [53]. However, the results described 
by Mermigkis et al. in 2012 showed that due to genetic and hormonal factors, there might be a delay in the normalization of the levels of this biomarker in women with OSA, requiring the use of CPAP for at least 6 months [54].

In addition to pro-inflammatory markers, studies describe the presence of prothrombotic markers in patients with OSA. Patients show higher circulating levels of plasminogen activator inhibitor (PAI)-1, an important endogenous inhibitor of fibrinolysis, resulting in increased atherothrombosis event risk, such as myocardial infarction (MI) and stroke. Soluble tissue factor (FTS) and von Willebrand factor (vWF) are also described in higher levels in patients with OSA, where FTS plays an important role in atherothrombosis due to exert procoagulant activity and propagation of thrombus after rupture atherosclerotic plaque. VWF plays procoagulant function by stabilizing the coagulation factor VIII and promoting platelet attachment at the site of atherosclerotic lesions, wherein the exposure of subendothelial structures occurs during MI [55, $56]$.

\section{Association studies of inflammatory response genes and OSAS}

Although much has been learned about the physiopathology and consequences of OSAS in recent years, the mechanisms and specific genes associated with these processes are still poorly known [57].

Petruco and Bagnato described in their study that a heritable component of OSAS has been recognized, but the elucidation of its genetic basis is difficult due to the heterogeneity of OSA phenotypes. The current paradigm was built as a product of intermediate phenotypes such as the craniofacial morphology, obesity, susceptibility to daytime sleepiness, ventilatory control, and upper airway control, which mutually interact and defines OSAS. However, it is not known which component of this product has a central role, and which appears as epiphenomena [57].

The IL-6, produced by different cells in the organism, is one of the most important mediators of inflammatory response and its role in the lipid metabolism and energy expenditure has already been described. According to Zhang et al., a polymorphism found in the promoter region of IL6 gene (-G174C) affects its gene expression. A similar role in the metabolism of the adipose tissue was also attributed to TNF- $\alpha$ where its levels also depend on the presence of a polymorphism (-G308A) in the promoter region of TNFA [58]. The presence of the A allele can double the expression of this gene, leading to a higher production of TNF- $\alpha$. Thus, it is possible to infer that the influence of the lipid metabolism and the energetic expenditure is linked to the concentration of these cytokines, which might contribute to weight gain in OSAS [42], being also a common risk factor for obesity and metabolic disturbances, especially insulin resistance [58].
According to Jones et.al., polymorphisms in the $C R P$ gene are associated with CRP serum concentrations. The authors evaluated the mean serum concentrations of CRP in healthy subjects and found that a specific genotype (CRP 1444C/T) is independently associated with increased high-sensitive CRP levels [59]. A study in North-American and Greek-European children pointed out that the change in IL-6 and CRP gene may affect susceptibility to OSA during childhood [60]. Also, other studies have identified associations between single nucleotide polymorphisms within CRP gene (rs2808630) and OSA in European Americans [56]. Kaditis et.al. also demonstrated that CRP (1444C/T and 1919A/T) and IL6 polymorphisms $(174 \mathrm{G} / \mathrm{C})$ were associated with an increased risk for the presence of OSA in the same population; however, such associations were identified in the Greek-European population. These findings suggest that variants of IL6 and CRP genes might also confer risk to the disease [60].

As previously described, TNF- $\alpha$ has also an important role in sleep regulation, since excessive daytime drowsiness, one of the main characteristics of OSAS consequences, is accompanied by increase in TNF- $\alpha$ levels. This relationship indicates that polymorphisms within this gene might also contribute to OSAS severity risk directly. Some studies have evaluated the association between the -G308A polymorphism within TNFA gene with TNF- $\alpha$ levels in OSAS patients. Riha et al. evaluated 206 individuals in the UK with $\mathrm{AHI} \geq 15$. As a result, it was observed that the presence of polymorphism resulted in the increase of the production of circulating TNF- $\alpha$ suggesting relationship with OSAS [61]. These results were corroborated by Bhushan et al. in 2009, who observed that Asian-Indian obese patients with OSAS showed higher frequency of the A allele of TNF- $\alpha(-308 \mathrm{~A})$ and that the levels of TNF- $\alpha$ directly correlated with the severity of OSAS, having as trigger mechanisms repetitive hypoxemic stress and sleep deprivation [62].

Due to the several association studies involving the TNFA308G/A polymorphism, Wu et al. have conducted a systematic review from 10 case-control studies evaluating the association between this polymorphism and OSAS [63]. The results of this review indicated that there is a significant association between this variation and OSAS, being A allele conferring higher risk in comparison to $\mathrm{G}$ allele $(\mathrm{OR}=1.67 ; 95 \%$ $\mathrm{CI}=1.43-1.95)$.

Other components related to the inflammatory response such as IL-6 have also been the target of genetic association studies. Larkin et al. genotyped six polymorphisms within the IL6 gene in Afro-American individuals and identified that a synonym variant in the coding region of the gene was significantly associated with decreased risk to OSAS, even after adjustment by body mass index. In that study, the authors suggest that the polymorphism in the IL- 6 gene can modulate the OSAS risk regardless of obesity [64].

Polymorphisms in genes that indirectly participate in processes related to inflammation, such as oxidative stress, were 
also studied in regard to their association with OSAS. An interesting study performed by Gozal et al. has shown that variations in the gene of the subunit pp22phox of the NADPH oxidase were associated with cognitive deficits in children with pediatric OSAS [65]. Moreover, other polymorphisms in this gene were also associated with OSAS in a case-control study, supporting its participation as an OSAS genetic risk factor [66].

\section{Gene expression studies of inflammatory response genes}

As the inflammatory process is activated in the pathogenesis of the respiratory disturbance present in OSAS, some studies have described the existence of gene expression changes of some cytokines [58]. Thus, a number of studies investigating how the physiological responses of OSAS can be consequences of transcriptional activation of inflammatory response genes have been performed to date.

As a consequence of apnea and hypopnea events in OSAS patients (sleep fragmentation, significant reduction of REM sleep and slow wave sleep, repetitive cycles of hypoxia/reoxygenation, and changes in the intrathoracic pressure), series of mechanisms related to inflammatory response can be activated [67]. For example, the activation of pro-inflammatory factors such as NF- $\mathrm{BB}$, and consequently, its related pathways were observed in chronic intermittent hypoxia conditions [68], possibly as a consequence of the increase in the production of ROS $[69,70]$. Moreover, regarding the damage related to sleep in patients with OSAS, it has been observed that the sympathetic activation associated to these events can also trigger an increase in the level of inflammatory markers as C-reactive protein (CRP), IL-6, and TNF- $\alpha$ [71, 72], a phenomenon also accompanied by an increase of IL- 6 and TNF- $\alpha$ mRNA in leukocytes [73]. An important factor that must be considered in the investigation of inflammatory response related to OSAS is the need to take into account confounder factor. For example, the coexistence of OSAS and obesity, besides the well-known relation between obesity and inflammation [74], and the interaction among hypoxia, inflammation, and adipose tissue [75] make the evaluation of this phenotype more complex. In this sense, the evaluation of OSAS patients with and without obesity becomes necessary in order to characterize the molecular components specific of each comorbidity.

More recent studies have investigated the expression of inflammatory response genes in target tissues such as the superior airway itself. Kimoff et al. have identified increased IL- 6 and TGF- $\beta$ mRNA expression in the superior airway mucosa followed by the increase of RANTES expression in the adjacent muscular tissue [76]. In animal samples, it was also observed that chronic intermittent hypoxia was capable of inducing a more pro-inflammatory response against allergens which might contribute to a more evident airflow limitation [77].
Studies investigating the gene expression of specific pathways related to OSAS have also been performed. Perry et al. investigated the expression profile of 84 genes related to hypoxia in controls and individuals with OSAS with different severity. Although genes directly related to inflammation were not studied, due to their well-known influence and activation of pro-inflammatory mechanisms as a consequence of hypoxia, a clear connection can be established. In this study, the authors found upregulation in specific genes related to hypoxia which had their expression regulated after treatment with CPAP in OSAS patients [78]. This approach has been little explored in OSAS, and it can offer a cost-effective alternative for further investigation of pathways in which a relationship with OSAS is already known, yet not fully characterized.

On the other hand, large-scale gene expression studies using microarrays have been frequently performed. Although without a priori hypothesis, these studies can identify potential new activated or repressed pathways which can be translated in novel physiopathological mechanisms. Using a systems biology approach, a field that integrates gene expression and computational algorithms in several levels to predict cell function as a whole, Liu et al. have identified networks of gene and protein interactions which suggest new molecular mechanisms associated to this pathology in adipose tissue. In some identified networks, molecules such as P38, MAPK, and STAT4, whose participation in inflammatory processes was a consequence of oxidative stress, were identified as important components in OSAS [79]. Another more recent study which also investigated the global gene expression in adipose tissue in patients with OSAS and controls identified sets of genes with increased expression in patients, including NF- $\mathrm{kB}$ proinflammatory pathways and pathways related to proteolysis [80]. These data suggest clear participation of inflammatory response in OSAS, with a hypothesis-free approach to OSAS gene expression investigation.

Besides gene expression studies, comprehensive investigations focused on the identification of epigenetic factors associated to OSAS are still lacking. Kim et al. aimed to find differences in the DNA methylation pattern, an important gene expression regulatory mechanism, of 24 inflammatory response genes in children with OSAS with and without high levels of ultra-sensitive CRP. In this study, DNA methylation in the promoter region of FOXP3, an important regulator of the expression of lymphocytes $\mathrm{T}$, was increased in individuals with higher levels of CRP, i.e., in those more likely to have increased systemic inflammatory response [81]. Studies investigating the microRNA expression profile, small RNAs which participate in the control of gene expression, are also lacking. In a study with rats, Zhang et al. identified that intermittent hypoxia followed by hypercapnia could decrease the expression of miR-34a [82], a miRNA associated to protection against tumorigenesis [83] and increase of the inflammatory cytokines expression (IL-6 and TNF- $\alpha$ ) [84]. 
The evidence shown here indicates that the inflammatory response is an important component in OSAS. However, the participation of this mechanism in patients who only presents mild OSAS has not yet been clarified. Often, the systemic and clinical alterations found in severe OSAS may not be detected in mild OSAS, although muscular alterations, that are much more sensitive to intermittent hypoxia mechanisms and oxidative stress can be present [67]. However, studies have not identified this hypothesis yet, suggesting an investigation opportunity not explored so far.

\section{Discussion}

Although much has been learned about the physiopathology and the consequences of OSAS in recent years, the mechanisms and the specific genes associated to these processes are still not completely known. Nevertheless, the documented data corroborated the hypothesis that mechanisms which involve inflammatory responses such as muscular alterations, drowsiness, insulin resistance, visceral obesity, and cardiovascular diseases are important to OSAS development and severity. Further studies aiming at the identification of mechanisms that regulate the gene expression changes in response to OSA are also warranted. Studies investigating how epigenetic regulation of inflammatory response by microRNAs, DNA methylation or histone modification is related to OSA still lack in the literature and are under development. Such dynamic mechanisms, when measured in accessible target tissues such as the blood, might help to identify specific groups of patients under higher risk of OSA or individuals with differential response to CPP treatment. Therefore, there is an urgent need for further and comprehensive studies aiming to investigate the gene expression of active inflammatory markers in mild OSAS, since this research field is underexplored and may provide a better and earlier treatment alternative for OSAS patients.

Acknowledgments This work was supported by grants from the Associação Fundo de Incentivo à Pesquisa (AFIP), Fundação de Amparo à Pesquisa do Estado de São Paulo, Coordenação de Aperfeiçoamento de Pessoal de Ensino Superior (CAPES), and Conselho Nacional de Desenvolvimento Científico e Tecnológico (CNPq).

Conflict of interest The authors declare that they have no competinginterests.

\section{References}

1. Azagra-Calero E, Espinar-Escalona E, Barrera-Mora J-M, et al. (2012) Obstructive sleep apnea syndrome (OSAS). Review of the literature. Med Oral Patol Oral Cir Bucal 17:e925-e929

2. Casale M, Pappacena M, Rinaldi V, et al. (2009) Obstructive sleep apnea syndrome: from phenotype to genetic basis. Curr Genomics 10:119-126. doi:10.2174/138920209787846998
3. Grilo A, Ruiz-Granados ES, Moreno-Rey C, et al. (2013) Genetic analysis of candidate SNPs for metabolic syndrome in obstructive sleep apnea (OSA). Gene 521:150-154. doi:10.1016/j.gene.2013.03.024

4. Sleep-related breathing disorders in adults: recommendations for syndrome definition and measurement techniques in clinical research (1999). The Report of an American Academy of Sleep Medicine Task Force. Sleep 22:667-689.

5. Garvey JF, Taylor CT, McNicholas WT (2009) Cardiovascular disease in obstructive sleep apnoea syndrome: the role of intermittent hypoxia and inflammation. Eur Respir J 33:1195-1205. doi:10. 1183/09031936.00111208

6. De Carvalho TBO, Suman M, Molina FD, et al. (2013) Relationship of obstructive sleep apnea syndrome with the 5HT2A receptor gene in Brazilian patients. Sleep Breath Schlaf Atm 17:57-62. doi:10.1007/s11325-012-0645-y

7. Young T, Palta M, Dempsey J, et al. (1993) The occurrence of sleep-disordered breathing among middle-aged adults. N Engl J Med 328:1230-1235. doi:10.1056/NEJM199304293281704

8. Peppard PE, Young T, Barnet JH, et al. (2013) Increased prevalence of sleep-disordered breathing in adults. Am J Epidemiol 177:10061014. doi:10.1093/aje/kws342

9. Tufik S, Santos-Silva R, Taddei JA, Bittencourt LRA (2010) Obstructive sleep apnea syndrome in the Sao Paulo Epidemiologic Sleep Study. Sleep Med 11:441-446. doi:10.1016/ j.sleep.2009.10.005

10. Young T, Peppard PE, Gottlieb DJ (2002) Epidemiology of obstructive sleep apnea: a population health perspective. Am J Respir Crit Care Med 165:1217-1239

11. Jordan AS, McSharry DG, Malhotra A (2014) Adult obstructive sleep apnoea. Lancet 383:736-747. doi:10.1016/S0140-6736(13) 60734-5

12. Schwartz AR, Patil SP, Laffan AM, et al. (2008) Obesity and obstructive sleep apnea: pathogenic mechanisms and therapeutic approaches. Proc Am Thorac Soc 5:185-192. doi:10.1513/pats. 200708-137MG

13. Peppard PE, Young T, Palta M, et al. (2000) Longitudinal study of moderate weight change and sleep-disordered breathing. JAMA 284:3015-3021

14. Ronen O, Malhotra A, Pillar G (2007) Influence of gender and age on upper-airway length during development. Pediatrics 120:e1028 e1034. doi:10.1542/peds.2006-3433

15. Whittle AT, Marshall I, Mortimore IL, et al. (1999) Neck soft tissue and fat distribution: comparison between normal men and women by magnetic resonance imaging. Thorax 54:323-328

16. Malhotra A, Huang Y, Fogel RB, et al. (2002) The male predisposition to pharyngeal collapse: importance of airway length. Am J Respir Crit Care Med 166:1388-1395. doi:10.1164/rccm.2112072

17. Young T (2001) Menopause, hormone replacement therapy, and sleep-disordered breathing: are we ready for the heat? Am J Respir Crit Care Med 163:597-598. doi:10.1164/ajrccm.163.3. ed09-01a

18. McNicholas WT (2009) Obstructive sleep apnea and inflammation. Prog Cardiovasc Dis 51:392-399. doi:10.1016/j.pcad.2008.10.005

19. Testelmans D, Tamisier R, Barone-Rochette G, et al. (2013) Profile of circulating cytokines: impact of OSA, obesity and acute cardiovascular events. Cytokine 62:210-216. doi:10.1016/j.cyto.2013.02.021

20. Ryan S, Taylor CT, McNicholas WT (2009) Systemic inflammation: a key factor in the pathogenesis of cardiovascular complications in obstructive sleep apnoea syndrome? Postgrad Med J 85: 693-698. doi:10.1136/thx.2008.105577

21. Lam S-Y, Liu Y, Ng K-M, et al. (2012) Chronic intermittent hypoxia induces local inflammation of the rat carotid body via functional upregulation of proinflammatory cytokine pathways. Histochem Cell Biol 137:303-317. doi:10.1007/s00418-011-0900-5

22. Grieve DJ, Shah AM (2003) Oxidative stress in heart failure. More than just damage. Eur Heart J 24:2161-2163 
23. Lavie L, Lavie P (2009) Molecular mechanisms of cardiovascular disease in OSAHS: the oxidative stress link. Eur Respir J 33:14671484. doi:10.1183/09031936.00086608

24. Pilkauskaite G, Miliauskas S, Sakalauskas R (2013) Reactive oxygen species production in peripheral blood neutrophils of obstructive sleep apnea patients. ScientificWorldJournal 2013:421763. doi: $10.1155 / 2013 / 421763$

25. Lavie L, Lavie P (2012) CrossTalk opposing view: most cardiovascular diseases in sleep apnoea are not caused by sympathetic activation. J Physiol 590:2817-2819. doi:10.1113/jphysiol.2012. 233833

26. Lui MM-S, Lam DC-L, Ip MS-M (2013) Significance of endothelial dysfunction in sleep-related breathing disorder. Respirol Carlton Vic 18:39-46. doi:10.1111/j.1440-1843.2012.02212.x

27. Flora Filho R, Zilberstein B (2000) Óxido nítrico: o simples mensageiro percorrendo a complexidade. Metabolismo, síntese e funções. Rev Assoc Médica Bras 46:265-271. doi:10.1590/ S0104-42302000000300012

28. Lavie L (2015) Oxidative stress in obstructive sleep apnea and intermittent hypoxia — revisited - the bad ugly and good: implications to the heart and brain. Sleep Med Rev 20:27-45. doi:10.1016/ j.smrv.2014.07.003

29. Hernández C, Abreu J, Abreu P, et al. (2006) Effects of nasal positive airway pressure treatment on oxidative stress in patients with sleep apnea-hypopnea syndrome. Arch Bronconeumol 42:125-129

30. Casella Filho A, Araújo RG, Galvão TG, Chagas ACP (2005) Inflamação e Aterosclerose: Integração de Novas Teorias e Valorização dos Novos Marcadores. Rev Bras Cardiol Invasiva 11:14-19

31. Kang WS, Park HJ, Chung J-H, Kim JW (2013) REM sleep deprivation increases the expression of interleukin genes in mice hypothalamus. Neurosci Lett 556:73-78. doi:10.1016/j.neulet. 2013.09.050

32. Krueger JM, Majde JA (2003) Humoral links between sleep and the immune system: research issues. Ann N Y Acad Sci 992:9-20

33. Yokoe T, Minoguchi K, Matsuo H, et al. (2003) Elevated levels of C-reactive protein and interleukin-6 in patients with obstructive sleep apnea syndrome are decreased by nasal continuous positive airway pressure. Circulation 107:1129-1134

34. Ohga E, Tomita T, Wada H, et al. (1985) (2003) Effects of obstructive sleep apnea on circulating ICAM-1, IL-8, and MCP-1. J Appl Physiol Bethesda Md 94:179-184. doi:10.1152/japplphysiol. 00177.2002

35. Ryan S, Taylor CT, McNicholas WT (2006) Predictors of elevated nuclear factor-kappaB-dependent genes in obstructive sleep apnea syndrome. Am J Respir Crit Care Med 174:824-830. doi:10.1164/ rccm.200601-066OC

36. Schulz R, Hummel C, Heinemann S, et al. (2002) Serum levels of vascular endothelial growth factor are elevated in patients with obstructive sleep apnea and severe nighttime hypoxia. Am J Respir Crit Care Med 165:67-70. doi:10.1164/ajrccm.165.1.2101062

37. Ip MS, Lam KS, Ho C, et al. (2000) Serum leptin and vascular risk factors in obstructive sleep apnea. Chest 118:580-586

38. Al Lawati N, Mulgrew A, Cheema R, et al. (2009) Proatherogenic cytokine profile of patients with suspected obstructive sleep apnea. Sleep Breath Schlaf Atm 13:391-395. doi:10. 1007/s11325-009-0259-1

39. Shamsuzzaman ASM, Winnicki M, Lanfranchi P, et al. (2002) Elevated C-reactive protein in patients with obstructive sleep apnea. Circulation 105:2462-2464

40. Guven SF, Turkkani MH, Ciftci B, et al. (2012) The relationship between high-sensitivity C-reactive protein levels and the severity of obstructive sleep apnea. Sleep Breath Schlaf Atm 16:217-221. doi:10.1007/s11325-011-0492-2
41. Quercioli A, Mach F, Montecucco F (2010) Inflammation accelerates atherosclerotic processes in obstructive sleep apnea syndrome (OSAS). Sleep Breath Schlaf Atm 14:261-269. doi:10.1007/ s11325-010-0338-3

42. Popko K, Gorska E, Potapinska O, et al. (2008) Frequency of distribution of inflammatory cytokines IL-1, IL-6 and TNF-alpha gene polymorphism in patients with obstructive sleep apnea. J Physiol Pharmacol Off J Pol Physiol Soc 59(Suppl 6):607-614

43. Semenza GL (1985) (2004) O2-regulated gene expression: transcriptional control of cardiorespiratory physiology by HIF-1. J Appl Physiol Bethesda Md 96:1173-1177 discussion 1170-1172. doi:10.1152/japplphysiol.00770.2003

44. Ryan S, Taylor CT, McNicholas WT (2005) Selective activation of inflammatory pathways by intermittent hypoxia in obstructive sleep apnea syndrome. Circulation 112:2660-2667. doi:10.1161/ CIRCULATIONAHA. 105.556746

45. Baessler A, Nadeem R, Harvey M, et al. (2013) Treatment for sleep apnea by continuous positive airway pressure improves levels of inflammatory markers - a meta-analysis. J Inflamm Lond Engl 10: 13. doi:10.1186/1476-9255-10-13

46. Nadeem R, Molnar J, Madbouly EM, et al. (2013) Serum inflammatory markers in obstructive sleep apnea: a meta-analysis. J Clin Sleep Med JCSM Off Publ Am Acad Sleep Med 9:1003-1012. doi: $10.5664 / j \mathrm{jsm} .3070$

47. Lui MM, Lam JC, Mak HK-F, et al. (2009) C-reactive protein is associated with obstructive sleep apnea independent of visceral obesity. Chest 135:950-956. doi:10.1378/chest.08-1798

48. Punjabi NM, Beamer BA (2007) C-reactive protein is associated with sleep disordered breathing independent of adiposity. Sleep 30:29-34

49. Guilleminault C, Kirisoglu C, Ohayon MM (2004) C-reactive protein and sleep-disordered breathing. Sleep 27:1507-1511

50. Mills PJ, Natarajan L, von Känel R, et al. (2009) Diurnal variability of C-reactive protein in obstructive sleep apnea. Sleep Breath Schlaf Atm 13:415-420. doi:10.1007/s11325-009-0268-0

51. Ishida K, Kato M, Kato Y, et al. (2009) Appropriate use of nasal continuous positive airway pressure decreases elevated C-reactive protein in patients with obstructive sleep apnea. Chest 136:125129. doi:10.1378/chest.08-1431

52. Xie X, Pan L, Ren D, et al. (2013) Effects of continuous positive airway pressure therapy on systemic inflammation in obstructive sleep apnea: a meta-analysis. Sleep Med 14:1139-1150. doi:10. 1016/j.sleep.2013.07.006

53. Guo Y, Pan L, Ren D, Xie X (2013) Impact of continuous positive airway pressure on $\mathrm{C}$-reactive protein in patients with obstructive sleep apnea: a meta-analysis. Sleep Breath Schlaf Atm 17:495-503. doi:10.1007/s11325-012-0722-2

54. Mermigkis C, Bouloukaki I, Mermigkis D, et al. (2012) CRP evolution pattern in CPAP-treated obstructive sleep apnea patients. Does gender play a role? Sleep Breath Schlaf Atm 16:813-819. doi:10.1007/s11325-011-0580-3

55. Von Känel R, Natarajan L, Ancoli-Israel S, et al. (2013) Effect of continuous positive airway pressure on day/night rhythm of prothrombotic markers in obstructive sleep apnea. Sleep Med 14: 58-65. doi:10.1016/j.sleep.2012.07.009

56. Larkin EK, Patel SR, Goodloe RJ, et al. (2010) A candidate gene study of obstructive sleep apnea in European Americans and African Americans. Am J Respir Crit Care Med 182:947-953. doi:10.1164/rccm.201002-0192OC

57. Petruco ACM, M da C B (2010) Aspectos genéticos da SAOS. J Bras Pneumol 36:13-16. doi:10.1590/S1806-37132010001400005

58. Zhang X, Liu R-Y, Lei Z, et al. (2009) Genetic variants in interleukin-6 modified risk of obstructive sleep apnea syndrome. Int J Mol Med 23:485-493

59. Jones J, Chen L-S, Baudhuin L, et al. (2009) Relationships between $\mathrm{C}$-reactive protein concentration and genotype in healthy 
volunteers. Clin Chem Lab Med CCLM FESCC 47:20-25. doi:10. 1515/CCLM.2009.005

60. Kaditis AG, Gozal D, Khalyfa A, et al. (2014) Variants in Creactive protein and IL- 6 genes and susceptibility to obstructive sleep apnea in children: a candidate-gene association study in European American and Southeast European populations. Sleep Med 15:228-235. doi:10.1016/j.sleep.2013.08.795

61. Riha RL, Brander P, Vennelle M, et al. (2005) Tumour necrosis factor-alpha (-308) gene polymorphism in obstructive sleep apnoea-hypopnoea syndrome. Eur Respir J 26:673-678. doi:10. 1183/09031936.05.00130804

62. Bhushan B, Guleria R, Misra A, et al. (2009) TNF-alpha gene polymorphism and TNF-alpha levels in obese Asian Indians with obstructive sleep apnea. Respir Med 103:386-392. doi:10.1016/j. rmed.2008.10.001

63. Wu Y, Cao C, Wu Y, et al. (2014) TNF- $\alpha$-308G/A polymorphism contributes to obstructive sleep apnea syndrome risk: evidence based on 10 case-control studies. PLoS One 9:e106183. doi:10. 1371/journal.pone.0106183

64. Larkin EK, Patel SR, Zhu X, et al. (2010) Study of the relationship between the interleukin- 6 gene and obstructive sleep apnea. Clin Transl Sci 3:337-339

65. Gozal D, Khalyfa A, Capdevila OS, et al. (2012) Cognitive function in prepubertal children with obstructive sleep apnea: a modifying role for NADPH oxidase p22 subunit gene polymorphisms? Antioxid Redox Signal 16:171-177. doi:10.1089/ars.2011.4189

66. Piérola J, Alemany A, Yañez A, et al. (2011) NADPH oxidase p22phox polymorphisms and oxidative stress in patients with obstructive sleep apnoea. Respir Med 105:1748-1754. doi:10.1016/j. rmed.2011.08.006

67. Arnardottir ES, Mackiewicz M, Gislason T, et al. (2009) Molecular signatures of obstructive sleep apnea in adults: a review and perspective. Sleep 32:447-470

68. Greenberg H, Ye X, Wilson D, et al. (2006) Chronic intermittent hypoxia activates nuclear factor-kappaB in cardiovascular tissues in vivo. Biochem Biophys Res Commun 343:591-596. doi:10. 1016/j.bbrc.2006.03.015

69. Lavie L (2005) Sleep-disordered breathing and cerebrovascular disease: a mechanistic approach. Neurol Clin 23:1059-1075. doi:10. 1016/j.ncl.2005.05.005

70. Lavie L (2003) Obstructive sleep apnoea syndrome - an oxidative stress disorder. Sleep Med Rev 7:35-51

71. Meier-Ewert HK, Ridker PM, Rifai N, et al. (2004) Effect of sleep loss on C-reactive protein, an inflammatory marker of cardiovascular risk. J Am Coll Cardiol 43:678-683. doi:10.1016/j. jacc.2003.07.050

72. Vgontzas AN, Zoumakis E, Bixler EO, et al. (2004) Adverse effects of modest sleep restriction on sleepiness, performance, and inflammatory cytokines. J Clin Endocrinol Metab 89:2119-2126. doi:10.1210/jc.2003-031562

73. Irwin MR, Wang M, Campomayor CO, et al. (2006) Sleep deprivation and activation of morning levels of cellular and genomic markers of inflammation. Arch Intern Med 166:1756-1762. doi: 10.1001/archinte.166.16.1756

74. Clement K, Langin D (2007) Regulation of inflammation-related genes in human adipose tissue. J Intern Med 262:422-430. doi:10. 1111/j.1365-2796.2007.01851.x

75. Taylor CT, Kent BD, Crinion SJ, et al. (2014) Human adipocytes are highly sensitive to intermittent hypoxia induced NFkappaB activity and subsequent inflammatory gene expression. Biochem Biophys Res Commun 447:660-665. doi:10.1016/j. bbrc.2014.04.062

76. Kimoff RJ, Hamid Q, Divangahi M, et al. (2011) Increased upper airway cytokines and oxidative stress in severe obstructive sleep apnoea. Eur Respir J 38:89-97. doi:10.1183/09031936.00048610

77. Broytman O, Braun RK, Morgan BJ, et al. (2014) Effects of chronic intermittent hypoxia on allergen-induced airway inflammation in rats. Am J Respir Cell Mol Biol. doi:10.1165/rcmb.2014-0213OC

78. Perry JC, Guindalini C, Bittencourt L, et al. (2013) Whole blood hypoxia-related gene expression reveals novel pathways to obstructive sleep apnea in humans. Respir Physiol Neurobiol 189:649654. doi:10.1016/j.resp.2013.08.012

79. Liu Y, Patel S, Nibbe R, et al. (2011) Systems biology analyses of gene expression and genome wide association study data in obstructive sleep apnea. Pac Symp Biocomput Pac Symp Biocomput 14-25

80. Gharib SA, Hayes AL, Rosen MJ, Patel SR (2013) A pathwaybased analysis on the effects of obstructive sleep apnea in modulating visceral fat transcriptome. Sleep 36:23-30. doi:10.5665/ sleep. 2294

81. Kim J, Bhattacharjee R, Khalyfa A, et al. (2012) DNA methylation in inflammatory genes among children with obstructive sleep apnea. Am J Respir Crit Care Med 185:330-338. doi:10.1164/rccm. 201106-1026OC

82. Zhang J, Guo X, Shi Y, et al. (2014) Intermittent hypoxia with or without hypercapnia is associated with tumorigenesis by decreasing the expression of brain derived neurotrophic factor and miR-34a in rats. Chin Med J 127:43-47

83. Liu C, Kelnar K, Liu B, et al. (2011) The microRNA miR-34a inhibits prostate cancer stem cells and metastasis by directly repressing CD44. Nat Med 17:211-215. doi:10.1038/nm.2284

84. Jiang P, Liu R, Zheng Y, et al. (2012) MiR-34a inhibits lipopolysaccharide-induced inflammatory response through targeting Notch1 in murine macrophages. Exp Cell Res 318: 1175-1184. doi:10.1016/j.yexcr.2012.03.018 